\title{
Effects of partial replacement of corn grain with lactose in calf starters on ruminal fermentation and growth performance
}

\author{
A. Saegusa, ${ }^{*}$ K. Inouchi, ${ }^{*} M$. Ueno, $†$ Y. Inabu,,$S$. Koike, $†$ T. Sugino, $\ddagger$ and M. Oba ${ }^{1}$ \\ *Dairy Technology Research Institute, Purchasing and Supplying Department, The National Federation of Dairy Co-operative Associations \\ (ZEN-RAKU-REN), Nishi-shirakawa, Fukushima, Japan 969-0223 \\ †Department of Animal Science, Hokkaido University, Sapporo, Japan 060-8589 \\ ‡Department of Bioresource Science, Hiroshima University, Higashi-Hiroshima, Japan 739-8528 \\ §Department of Animal, Food and Nutritional Science, University of Alberta, Edmonton, Canada T6G 2P5
}

\begin{abstract}
The objective of this study was to evaluate effects of partial replacement of dry ground corn with lactose in calf starters on dry matter intake, growth rate, ruminal $\mathrm{pH}$, and volatile fatty acid profile. Sixty Holstein bull calves were raised on a high plane of nutrition program until $55 \mathrm{~d}$ of age. Calves were fed texturized calf starters containing $30.1 \%$ steam-flaked grains and lactose at 0 (control), 5, or $10 \%$ ( $\mathrm{n}=20$ for each treatment) on a dry matter basis. All calves were fed treatment calf starters ad libitum from d 7 and kleingrass hay from d 35. Ruminal $\mathrm{pH}$ was measured continuously immediately after weaning (d 55-62) for 15 calves $(\mathrm{n}=5$ per treatment), and $3 \mathrm{wk}$ after weaning (d 77 to 80 ) for the other 45 calves ( $\mathrm{n}=15$ per treatment). Dry matter intake, growth performance, and ruminal $\mathrm{pH}$ variables were not affected by treatment. However, according to Spearman's correlation coefficient $\left(r_{s}\right)$ analyses, lactose intake was positively correlated with dairy minimum ruminal $\mathrm{pH}\left(r_{s}=0.306\right)$ for the data collected from d 77 to 80 . Similarly, hay intake was not affected by treatment, but positively correlated with daily mean $\left(r_{s}=0.338\right)$ and maximum ruminal $\mathrm{pH}\left(r_{s}=0.408\right)$ and negatively correlated with duration $\mathrm{pH}<5.8\left(r_{s}\right.$ $=-0.329)$ and area $\mathrm{pH}<5.8\left(r_{s}=-0.325\right)$, indicating that the variation in hay intake among animals might have masked treatment effects on ruminal $\mathrm{pH}$. Ruminal molar ratio of acetate was higher (45.2 vs. $40.6 \%$ ), and that of propionate was lower in $10 \%$ lactose than control (35.3 vs. 40.2\%) for ruminal fluid collected on d 80; however, molar ratio of butyrate was not affected by treatment. These results indicate that lactose inclusion in calf starters up to $10 \%$ of dry matter might not affect
\end{abstract}

Received January 19, 2017.

Accepted May 3, 2017.

${ }^{1}$ Corresponding author: moba@ualberta.ca dry matter intake and growth performance of calves, but that greater lactose and hay intake might be associated with higher ruminal $\mathrm{pH}$.

Key words: calf, lactose, starter, volatile fatty acids, ruminal $\mathrm{pH}$

\section{INTRODUCTION}

Consumption of easily fermentable carbohydrates in calf starters can stimulate rumen development and growth of epithelium of rumen (Baldwin et al., 2004; Drackley, 2008), microbial proliferation (Yáñez-Ruiz et al., 2015), and VFA production (Suárez et al., 2006a; Khan et al., 2016), as well as increased propionate and butyrate production in the rumen (Tamate et al., 1962; Khan et al., 2016). Dietary composition of calf starter can affect ruminal epithelial development by altering microbial fermentation end products (Nocek et al., 1984; Khan et al., 2008; Suárez et al., 2006b), and butyrate is considered to stimulate rumen epithelial growth to a greater extent than the other VFA (Tamate et al., 1962; Bergman, 1990).

However, high-starch diets often induce low ruminal pH in calves (Suárez et al., 2006a; Khan et al., 2016). During the weaning transition, calves often experienced ruminal pH below 5.8 (Anderson et al., 1987), which is attributed to large intake of rapidly fermentable carbohydrates (Khan et al., 2016). In addition, the low ruminal $\mathrm{pH}$ might be also attributed to underdeveloped ruminal epithelium of calves, where fermentation acid production exceeds the absorptive capacity of the ruminal wall (Williams et al., 1987). Subacute ruminal acidosis, defined as ruminal pH below 5.8 (Garret, 1996), is associated with depressed DMI, laminitis, and rumenitis in mature cows (Kleen et al., 2003), and decreased rumen motility and increased keratinization of the papillae in calves (Bull et al., 1965). In addition, SARA can cause liver abscesses, as low ruminal pH can damage rumen wall allowing pyogenic bacteria to reach the liver (Kay, 1960; Bull et al., 1965). 
Feeding lactose, the primary nutrient in whey, may mitigate SARA in calves. Chamberlain et al. (1993) reported that feeding lactose increased ruminal $\mathrm{pH}$ in sheep compared with other sugars and starch. Dietary inclusion of lactose (DeFrain et al., 2004, 2006) or a ruminal dose of lactose (Oba et al., 2015) increased ruminal butyrate concentration in mature cows. In addition, lactose feeding tended to increase DMI in mature cows (DeFrain et al., 2004); however, effects of feeding lactose on ruminal fermentation and animal performance have not been extensively studied for calves.

We hypothesized that lactose inclusion in calf starters would increase ruminal butyrate concentration, ruminal $\mathrm{pH}$, starter intake, and growth performance of calves. The objective of our study was to evaluate effects of partial replacement of a starch source with lactose in calf starters on DMI and growth performance before and after weaning, as well as ruminal $\mathrm{pH}$ and VFA profiles after weaning.

\section{MATERIALS AND METHODS}

\section{Animals and Housing}

Sixty Holstein male calves (4-6 d of age, BW $=47.3$ $\pm 0.7 \mathrm{~kg}$; mean $\pm \mathrm{SD}$ ) were collected from commercial dairies in Fukushima and Ibaraki prefectures (Japan) and transported to the Dairy Technology Research Institute (Yabuki, Fukushima, Japan). Calves were born on March 20 to April 13, 2015 (group 1), and May 7 to June 2, 2015 (Group 2). Calves were further blocked by birthdate, BW, and farm origin, and randomly assigned to 1 of 3 calf starter treatments $(n=20$ for each treatment). Calves were raised in individual hatches (made by fiber-reinforced plastics with wood grating floor) without bedding materials. When calves were arrived in the research farm, they received $5 \mathrm{~mL}$ of Terramycin (Zoetis Japan, Tokyo, Japan) and $0.1 \mathrm{~mL}$ of Duphafral Forte (Zoetis Japan) via subcutaneous injection and received $5 \mathrm{~mL}$ of Ivermec PO (Fujita Pharm, Tokyo, Japan) via percutaneous absorption. In addition, all calves received $5 \mathrm{~mL}$ of Ektecin Liquid (Meiji Seika Pharma, Tokyo, Japan) and $20 \mathrm{~mL}$ of Baycox Bovis (Bayer Yakuhin, Osaka, Japan) via oral administration on d 3 and 21 after arrival, respectively.

\section{Feeding}

All calves were fed a milk replacer $(28 \% \mathrm{CP}$ and $15 \%$ fat; $166.7 \mathrm{~g} / \mathrm{L}$ ) using a bucket with a soft rubber nipple twice daily at 0615 and $1615 \mathrm{~h}$. Milk replacer was offered at $600 \mathrm{~g} / \mathrm{d}$ until d 13, $800 \mathrm{~g} / \mathrm{d}$ from d 14 to 20 , and
$1,200 \mathrm{~g} / \mathrm{d}$ from d 21 to $41,800 \mathrm{~g} / \mathrm{d}$ from d 42 to 48 , and $600 \mathrm{~g} / \mathrm{d}$ from d 49 to 55 ; calves were then weaned on d 56. All calves had free access to fresh water supplied by a bucket with a soft rubber nipple. Calves were fed texturized calf starters containing $30.1 \%$ steam-flaked grains and lactose at 0 (control), 5.0 (LAC5), or $10.0 \%$ (LAC10) on a DM basis. All calf starters were formulated for 23.1\% CP (Table 1). Treatment calf starters were offered ad libitum using an 8-L bucket from d 7 . Feeding time of calf starters was $1000 \mathrm{~h}$ initially, but when calves consumed more than $900 \mathrm{~g} / \mathrm{d}$ (as fed) of starter, calves were fed twice daily (1000 and $1500 \mathrm{~h}$; equal volume of starter). Kleingrass hay was offered at $50 \mathrm{~g} / \mathrm{d}$ (as fed) from d 42 to $48,100 \mathrm{~g} / \mathrm{d}$ (as fed) from d 49 to 55 , and $150 \mathrm{~g} / \mathrm{d}$ (as fed) after d 56. Refused calf starters and hay were cleaned daily at $1000 \mathrm{~h}$ and their intakes were recorded.

\section{Data and Sample Collection}

Body weight, withers height, hip height, horizontal body length, hip width, and heart girth were measured

Table 1. Dry matter ratio of ingredients on calf starter formulations

\begin{tabular}{|c|c|c|c|}
\hline \multirow[b]{2}{*}{ Composition } & \multicolumn{3}{|c|}{ Treatment $^{1}$} \\
\hline & Control & LAC5 & LAC10 \\
\hline \multicolumn{4}{|l|}{ Ingredient, $\%$ of DM } \\
\hline Steam-flaked corn grain & 9.9 & 9.9 & 9.9 \\
\hline Steam-flaked barley grain & 20.2 & 20.2 & 20.2 \\
\hline Alfalfa dehydrated pellet & 3.7 & 3.7 & 3.7 \\
\hline Molasses cane & 0.4 & 0.4 & 0.4 \\
\hline Pellet & 65.8 & 65.8 & 65.8 \\
\hline \multicolumn{4}{|l|}{ Pellet, \% of DM } \\
\hline Dry ground corn & 14.9 & 8.2 & 1.6 \\
\hline Wheat feed flour & 1.6 & 1.6 & 1.6 \\
\hline Soybean flour & 2.2 & 3.4 & 4.4 \\
\hline Wheat bran & 9.0 & 9.0 & 9.0 \\
\hline Soybean meal & 17.3 & 16.8 & 14.6 \\
\hline Rapeseed meal & 1.3 & 1.3 & 1.3 \\
\hline Heated soybean $^{2}$ & 7.1 & 7.1 & 7.1 \\
\hline Corn gluten meal & 2.3 & 2.6 & 4.1 \\
\hline Ground beet pulp & 4.1 & 4.1 & 4.1 \\
\hline Dehydrated alfalfa & 0.0 & 0.6 & 1.9 \\
\hline Cane molasses & 3.7 & 3.7 & 3.7 \\
\hline Calcium carbonate & 1.2 & 1.2 & 1.2 \\
\hline Salt & 0.7 & 0.7 & 0.7 \\
\hline Calcium phosphate & 0.6 & 0.6 & 0.6 \\
\hline $\mathrm{GC} \operatorname{mix} 21^{3}$ & 0.5 & 0.5 & 0.5 \\
\hline Lactose $^{4}$ & 0.0 & 5.0 & 10.0 \\
\hline
\end{tabular}

${ }^{1}$ Treatment: Control $=$ calf starter containing no lactose; LAC5 = calf starter containing $5 \%$ of lactose on a DM basis; LAC10 $=$ calf starter containing $10 \%$ of lactose on a DM basis.

${ }^{2}$ Heated soybean (SoyPlus, West Central Cooperative, Ralston, IA).

${ }^{3} \mathrm{GC}$ mix 21 (trace mineral and vitamin premix, Zenrakuren, Tokyo, Japan), containing vitamin mix $16.0 \%$, trace mineral mix $6.3 \%$, and rice bran $77.7 \%$.

${ }^{4}$ Lactose (Hilmar 5030 Extra Fine Grind Lactose, Hilmar Ingredients, Hilmar, CA). 
at the start of trial (d 7) and weekly thereafter until the end of trial (d 80). Fecal score (1-4 scales; $1=$ normal fecal consistency to $4=$ severe diarrhea) and diseases incidences, if any, were recorded daily. Blood was sampled from a jugular vein on $\mathrm{d} 7$ after birth and serum was harvested. Ruminal $\mathrm{pH}$ was measured using Small Ruminal pH Data Loggers (SRL T-9, DASCOR, Escondido, CA) every 2 min from d 55 to 62, immediately after weaning, for 15 calves ( $\mathrm{n}=5$ per treatment), and from d 77 to 80, 3 wk after weaning, for the other 45 calves ( $\mathrm{n}=15$ per treatment), as described by Laarman et al. (2012). All pH probes were calibrated at $\mathrm{pH}$ 4 and 7 before and after ruminal $\mathrm{pH}$ measurements. Mean, minimum, and maximum $\mathrm{pH}$ values as well as duration and area under $\mathrm{pH} 5.8$ were calculated daily and averaged.

Fifteen calves ( $\mathrm{n}=5$ per treatment) were euthanized on $\mathrm{d} 62$, and the other 45 calves $(\mathrm{n}=15$ per treatment) were euthanized on d 80. After BW was measured, calves were anesthetized (subcutaneous injection of Selactar $2 \%$ injection solution at $1.5 \mathrm{~mL} / \mathrm{kg}$ of $\mathrm{BW}$; Bayer Yakuhin) and killed by exsanguination from the carotid artery. Ruminal fluid $(50 \mathrm{~mL})$ was sampled immediately after euthanization and frozen at $-20^{\circ} \mathrm{C}$ until further analysis. Digestive organs were weighed and rumen papillae were sampled to determine expression of selected genes, and these data were reported elsewhere (Inabu et al., 2016).

\section{Sample Analysis}

Treatment calf starters were sampled weekly, composited monthly, and stored at room temperature. The samples were ground using a hammer mill (SM1, Retsch GmbH, Haan Germany) with a 1-mm screen, and analyzed by Zen-Raku-Ren Analysis Center (Kamisu, Ibaraki, Japan) for concentrations of DM, ash, $\mathrm{CP}$, ether extract, and starch according to AOAC (1990), and for NDF and ADF according to AOAC International (2002). Lactose content was analyzed by a commercial laboratory (Japan Food Research Laboratories, Tokyo, Japan) using HPLC (LC-20AD, Shimadzu, Kyoto, Japan) according to Government of Japan (2015). Ethanol-soluble carbohydrate concentration was analyzed by Cumberland Valley Analytical Services (Hagerstown, MD) according to Hall et al. (1999). Serum samples were analyzed for IgG concentration by single radial immunodiffusion method using a commercial kit (Bovine IgG SRID assay kit LL-70002, Life laboratory, Yamagata, Japan). Ruminal VFA profile was analyzed using gas chromatography (GC14B, Shimadzu) according to the method described by Watanabe et al. (2010).

\section{Statistical Analysis}

All response variables except for ruminal $\mathrm{pH}$ and VFA profile were analyzed separately for 3 phases differing in primary nutrient sources; before weaning (d 7-41), during weaning transition (d 42-55), and after weaning (d 56-80). In addition, as calves were purchased at 2 different periods, group effect and group by treatment interaction were included then statistical model to account for possible confounding effects of different environment to which calves were exposed. Data were analyzed using JMP 12 (SAS Institute Inc., Cary, NC) according to the following model:

$$
\begin{aligned}
Y_{\mathrm{ijk}}= & \mu+\mathrm{T}_{\mathrm{i}}+\mathrm{W}_{\mathrm{j}}+\mathrm{G}_{\mathrm{k}}+\mathrm{TW}_{\mathrm{ij}} \\
& +\mathrm{TG}_{\mathrm{ik}}+\mathrm{Cov}+\mathrm{e}_{\mathrm{ijk}},
\end{aligned}
$$

where $Y_{\mathrm{ijk}}$ is the dependent variable, $\mu$ is overall mean, $T_{i}$ is fixed effect of treatment, $W_{j}$ is the fixed effect of week used as a repeated measure, $\mathrm{G}_{\mathrm{k}}$ is the fixed effect of group, $\mathrm{TW}_{\mathrm{ij}}$ is the effect of treatment by week interaction, $\mathrm{TG}_{\mathrm{ik}}$ is the effect of treatment by group interaction, $\mathrm{Cov}$ is the $\mathrm{IgG}$ concentration of serum samples collected on wk 1 used as covariate, and $\mathrm{e}_{\mathrm{ijk}}$ is the residual. Treatment effects were declared significant at $P<0.05$ and tendencies were declared at 0.05 $\leq P<0.10$.

Ruminal $\mathrm{pH}$ and VFA data were analyzed using JMP 12 (SAS Institute Inc.) according to the following model:

$$
\mathrm{Y}_{\mathrm{ijk}}=\mu+\mathrm{T}_{\mathrm{i}}+\mathrm{G}_{\mathrm{k}}+\mathrm{TG}_{\mathrm{ik}}+\operatorname{Cov}+\mathrm{e}_{\mathrm{ik}},
$$

where $Y_{\mathrm{ijk}}$ is the dependent variable, $\mu$ is overall mean, $T_{i}$ is fixed effect of treatment, $G_{k}$ is the fixed effect of group, $\mathrm{TG}_{\mathrm{ik}}$ is the effect of treatment by group interaction, Cov is the IgG concentration of plasma samples collected on wk 1 used as covariate, and $\mathrm{e}_{\mathrm{ik}}$ is the residual. Treatment effects were declared significant at $P<0.05$ and tendencies were declared at $0.05 \leq P<$ 0.10 . Correlations of ruminal $\mathrm{pH}$ variables to DMI, calf starter intake, hay intake, starch intake, and lactose intake were analyzed by Spearman's correlation method of JMP 12 (SAS Institute Inc.).

\section{RESULTS}

One calf in the LAC5 treatment had severe pneumonia and another calf in LAC10 treatment had severe arthritis, these calves were excluded from statistical analysis. Furthermore, ruminal $\mathrm{pH}$ data were missing for 1 calf each for the control and LAC10 treatments 
Table 2. Nutrient composition of treatment calf starters (mean $\pm \mathrm{SD}$ )

\begin{tabular}{|c|c|c|c|}
\hline \multirow[b]{2}{*}{ Item } & \multicolumn{3}{|c|}{ Treatment $^{1}$} \\
\hline & Control & LAC5 & LAC10 \\
\hline $\begin{array}{l}\mathrm{DM}, \% \\
\text { Nutrient component, \% of DM }\end{array}$ & $89.7 \pm 0.1$ & $89.6 \pm 0.2$ & $90.0 \pm 0.5$ \\
\hline CP & $24.0 \pm 0.4$ & $23.3 \pm 1.0$ & $24.4 \pm 0.8$ \\
\hline Ether extract & $3.5 \pm 0.2$ & $4.3 \pm 0.1$ & $4.2 \pm 0.2$ \\
\hline Ash & $6.5 \pm 0.2$ & $6.8 \pm 0.3$ & $7.4 \pm 1.4$ \\
\hline $\mathrm{NDF}$ & $16.5 \pm 0.9$ & $16.6 \pm 0.5$ & $17.6 \pm 0.6$ \\
\hline $\mathrm{ADF}$ & $7.9 \pm 0.5$ & $8.3 \pm 0.2$ & $9.0 \pm 0.2$ \\
\hline $\mathrm{ESC}^{2}$ & $10.3 \pm 0.6$ & $11.7 \pm 0.7$ & $15.8 \pm 0.7$ \\
\hline Starch & $29.7 \pm 1.1$ & $28.1 \pm 2.3$ & $21.9 \pm 1.1$ \\
\hline Lactose & $0.0 \pm 0.0$ & $3.0 \pm 0.4$ & $7.2 \pm 0.2$ \\
\hline
\end{tabular}

due to the failure of small ruminant ruminal $\mathrm{pH}$-logger system.

Analyzed nutrient composition of calf starters is shown in Table 2. Starch concentration was 29.7, 28.1, and $21.9 \%$ and lactose concentration was $0,3.0$, and $7.2 \%$ for control, LAC5, and LAC10, on a DM basis, respectively. Analyzed lactose concentration was lower than formulated values possibly because of the Maillard reaction from the pelleting procedure.

Dry matter intake, starter intake, and hay intake were not affected by treatment (Table 3). Starch intake was lower for calves fed LAC5 and LAC10 than those fed control before weaning ( 32.2 and 30.0 vs. $46.0 \mathrm{~g} / \mathrm{d}$; $P<0.05)$ and during the weaning transition (174.4 and 168.8 vs. $231.5 \mathrm{~g} / \mathrm{d} ; P<0.05)$, respectively. In addition, starch intake after weaning was different among all treatments (822.7, 739.1, and $616.5 \mathrm{~g} / \mathrm{d}$ for control, LAC5, and LAC10, respectively; $P<0.05)$. Lactose intake was different among all treatment before weaning $(0.0,3.5$, and $9.8 \mathrm{~g} / \mathrm{d}$ for control, LAC5, and LAC10, respectively; $P<0.05)$, during weaning transition $(0.0$, 18.5 , and $55.6 \mathrm{~g} / \mathrm{d}$ for control, LAC5, and LAC10, respectively; $P<0.05$ ), and after weaning $(0.0,78.4$, and $201.6 \mathrm{~g} / \mathrm{d}$ for control, LAC5, and LAC10, respectively; $P<0.05)$. Intake of NDF was higher in calves fed LAC10 than those fed control and LAC5 (544.2 vs. 505.4 and $487.1 \mathrm{~g} / \mathrm{d} ; P<0.05)$ after weaning. Treatment did not affect ADG and other growth variables (Table 4); in addition, fecal score was not different among treatments (Table 5).

Ruminal $\mathrm{pH}$ immediately after weaning or $3 \mathrm{wk}$ after weaning were not affected by treatment (Table 6 ); however, lactose intake was positively correlated $(P<0.05)$ to daily minimum ruminal $\mathrm{pH}$ [Spearman's correlation coefficient $\left.\left(\boldsymbol{r}_{s}\right)=0.306\right]$ and tended to be negatively correlated $(P<0.10)$ with area $\mathrm{pH}<5.8\left(r_{s}=-0.268\right)$ at 3 wk after weaning (Table 7 ). In addition, hay intake was positively correlated $(P<0.05)$ to daily mean $\mathrm{pH}$ $\left(r_{s}=0.408\right)$ and maximum ruminal $\mathrm{pH}\left(r_{s}=0.338\right)$, and negatively correlated $(P<0.05)$ to duration $\mathrm{pH}$ $<5.8\left(r_{s}=-0.329\right)$ and area $\mathrm{pH}<5.8\left(r_{s}=-0.325\right)$ at 3 wk after weaning.

Molar ratios of ruminal acetate, propionate, and butyrate were not affected by treatment at 1 wk after weaning (Table 8). However, calves fed LAC10 had higher molar ratio of acetate $(45.2$ vs. $40.6 \% ; P<0.05)$ and lower molar ratio of propionate $(35.3$ vs. $40.2 \% ; P$ $<0.05)$ compared with those fed control at 3 wk after weaning. Consequently, acetate-to-propionate ratio was higher in calves fed LAC10 than those fed control (1.29 vs. 1.04; $P<0.05$ ); however, the molar ratio of ruminal butyrate was not affected by treatment.

\section{DISCUSSION}

Calves during the weaning transition often experience ruminal pH below pH5.8 (Anderson et al., 1987; Quigley et al., 1992a; Laarman et al., 2012), which may decrease DMI (Khan et al., 2008). Therefore, we hypothesized that we would be able to increase calf starter intake and growth performance if we prevent SARA in calves. We had expected that partial replacement of corn grain with lactose in calf starters would increase ruminal pH of calves, as Chamberlain et al. (1993) reported that addition of lactose to a basal diet in sheep (silage only) resulted in higher ruminal $\mathrm{pH}$ compared with addition of other carbohydrates (lactose, xylose, starch, and fructose). Previous studies that evaluated effects of feeding lactose to mature cows (DeFrain et al., 2004; DeFrain et al., 2006; Oba et al., 2015) or sheep (Chamberlain et al., 1993) reported increased ruminal butyrate concentration. Because greater butyrate production in the rumen is expected to decrease proton production per unit of OM fermentation compared 
Table 3. Effects of feeding calf starters differing in lactose content on feed and nutrient intakes before weaning (d 7-41), during weaning transition (d 42-55), and after weaning (d 56-80; LSM \pm SEM)

\begin{tabular}{|c|c|c|c|c|}
\hline \multirow[b]{2}{*}{ Item } & \multicolumn{3}{|c|}{ Treatment $^{1}$} & \multirow[b]{2}{*}{$P$-value } \\
\hline & $\begin{array}{c}\text { Control } \\
(\mathrm{n}=15)\end{array}$ & $\begin{array}{c}\text { LAC5 } \\
(\mathrm{n}=14)\end{array}$ & $\begin{array}{c}\text { LAC10 } \\
(\mathrm{n}=14)\end{array}$ & \\
\hline \multicolumn{5}{|c|}{ Before weaning (d 7-41) } \\
\hline Total DMI, ${ }^{2} \mathrm{~g} / \mathrm{d}$ & $1,117.1 \pm 15.78$ & $1,080.0 \pm 20.08$ & $1,101.5 \pm 16.34$ & 0.35 \\
\hline Starter DMI, $\mathrm{g} / \mathrm{d}$ & $154.9 \pm 12.84$ & $115.0 \pm 16.44$ & $136.6 \pm 13.30$ & 0.16 \\
\hline Starch intake, g/d & $46.0 \pm 3.61^{\mathrm{a}}$ & $32.2 \pm 4.62^{\mathrm{b}}$ & $30.0 \pm 3.74^{\mathrm{b}}$ & 0.01 \\
\hline Lactose intake, $\mathrm{g} / \mathrm{d}$ & $0.0 \pm 0.43^{\mathrm{c}}$ & $3.5 \pm 0.55^{\mathrm{b}}$ & $9.8 \pm 0.43^{\mathrm{a}}$ & $<0.01$ \\
\hline NDF intake, g/d & $25.6 \pm 2.15$ & $19.1 \pm 2.75$ & $24.0 \pm 2.23$ & 0.17 \\
\hline \multicolumn{5}{|c|}{ Weaning transition $(\mathrm{d} 42-55)$} \\
\hline Total DMI, ${ }^{2} \mathrm{~g} / \mathrm{d}$ & $1,487.2 \pm 64.86$ & $1,336.3 \pm 75.50$ & $1,485.8 \pm 67.55$ & 0.24 \\
\hline Starter DMI, g/d & $778.2 \pm 64.87$ & $621.1 \pm 75.30$ & $770.0 \pm 67.66$ & 0.23 \\
\hline Hay DMI, g/d & $33.5 \pm 2.29$ & $38.9 \pm 2.89$ & $39.1 \pm 2.37$ & 0.18 \\
\hline Starch intake, g/d & $231.5 \pm 18.26^{\mathrm{a}}$ & $174.4 \pm 21.20^{\mathrm{b}}$ & $168.8 \pm 19.02^{\mathrm{b}}$ & 0.03 \\
\hline Lactose intake, g/d & $0.0 \pm 2.16^{\mathrm{c}}$ & $18.5 \pm 2.57^{\mathrm{b}}$ & $55.6 \pm 2.25^{\mathrm{a}}$ & $<0.01$ \\
\hline NDF intake, $\mathrm{g} / \mathrm{d}$ & $150.1 \pm 10.93$ & $128.9 \pm 11.32$ & $161.7 \pm 10.94$ & 0.16 \\
\hline \multicolumn{5}{|c|}{ After weaning (d 56-80) } \\
\hline Total DMI ${ }^{2} \mathrm{~g} / \mathrm{d}$ & $2,843.3 \pm 68.68$ & $2,710.3 \pm 73.26$ & $2,885.3 \pm 71.34$ & 0.21 \\
\hline Starter DMI, g/d & $2,768.9 \pm 68.24$ & $2,633.7 \pm 71.98$ & $2,809.3 \pm 70.93$ & 0.19 \\
\hline Hay DMI, g/d & $75.1 \pm 4.57$ & $77.6 \pm 5.06$ & $77.1 \pm 4.74$ & 0.92 \\
\hline Starch intake, $\mathrm{g} / \mathrm{d}$ & $822.7 \pm 19.11^{\mathrm{a}}$ & $739.1 \pm 20.19^{\mathrm{b}}$ & $616.5 \pm 19.87^{\mathrm{c}}$ & $<0.01$ \\
\hline Lactose intake, $\mathrm{g} / \mathrm{d}$ & $0.0 \pm 2.32^{\mathrm{c}}$ & $78.4 \pm 2.43^{\mathrm{b}}$ & $201.6 \pm 2.42^{\mathrm{a}}$ & $<0.01$ \\
\hline NDF intake, g/d & $505.4 \pm 11.99^{\mathrm{b}}$ & $487.1 \pm 13.06^{\mathrm{b}}$ & $544.2 \pm 12.45^{\mathrm{a}}$ & 0.01 \\
\hline
\end{tabular}

${ }^{\mathrm{a}-\mathrm{c}}$ Means within a row differ $(P<0.05)$ if superscript letters differ.

${ }^{1}$ Treatment: control $=$ calf starter containing no lactose; LAC5 $=$ calf starter containing $5 \%$ of lactose on a DM basis; $\mathrm{LAC} 10=$ calf starter containing $10 \%$ of lactose on a DM basis.

${ }^{2}$ Total DMI is a sum of milk replacer, starter, and hay intakes.

Table 4. Effects of feeding calf starters differing in lactose content on growth performance before weaning (d 7-41), during weaning transition (d 42-55), and after weaning (d 56-80; LSM \pm SEM)

\begin{tabular}{|c|c|c|c|c|}
\hline \multirow[b]{2}{*}{ Item } & \multicolumn{3}{|c|}{ Treatment ${ }^{1}$} & \multirow[b]{2}{*}{$P$-value } \\
\hline & $\begin{array}{l}\text { Control } \\
(\mathrm{n}=15)\end{array}$ & $\begin{array}{c}\text { LAC5 } \\
(\mathrm{n}=14)\end{array}$ & $\begin{array}{c}\text { LAC10 } \\
(\mathrm{n}=14)\end{array}$ & \\
\hline \multicolumn{5}{|l|}{ Before weaning (d 7-41) } \\
\hline $\mathrm{ADG}, \mathrm{kg} / \mathrm{d}$ & $0.78 \pm 0.042$ & $0.73 \pm 0.043$ & $0.79 \pm 0.043$ & 0.50 \\
\hline Withers height gain, $\mathrm{cm} / \mathrm{d}$ & $0.24 \pm 0.018$ & $0.22 \pm 0.018$ & $0.24 \pm 0.019$ & 0.69 \\
\hline Hip height gain, cm/d & $0.22 \pm 0.025$ & $0.24 \pm 0.026$ & $0.23 \pm 0.025$ & 0.94 \\
\hline Body length gain, $\mathrm{cm} / \mathrm{d}$ & $0.33 \pm 0.028$ & $0.25 \pm 0.028$ & $0.30 \pm 0.029$ & 0.11 \\
\hline Heart girth gain, $\mathrm{cm} / \mathrm{d}$ & $0.40 \pm 0.037$ & $0.38 \pm 0.037$ & $0.41 \pm 0.038$ & 0.80 \\
\hline Hip width gain, $\mathrm{cm} / \mathrm{d}$ & $0.09 \pm 0.011$ & $0.09 \pm 0.011$ & $0.09 \pm 0.011$ & 0.99 \\
\hline ADG/total DMI, $\mathrm{kg} / \mathrm{kg}$ & $0.67 \pm 0.041$ & $0.65 \pm 0.041$ & $0.69 \pm 0.043$ & 0.83 \\
\hline \multicolumn{5}{|l|}{ Weaning transition (d 42-55) } \\
\hline $\mathrm{ADG}, \mathrm{kg} / \mathrm{d}$ & $0.74 \pm 0.061$ & $0.69 \pm 0.065$ & $0.86 \pm 0.063$ & 0.17 \\
\hline Withers height gain, $\mathrm{cm} / \mathrm{d}$ & $0.21 \pm 0.028$ & $0.26 \pm 0.029$ & $0.25 \pm 0.029$ & 0.52 \\
\hline Hip height gain, cm/d & $0.27 \pm 0.031$ & $0.26 \pm 0.020$ & $0.26 \pm 0.032$ & 0.96 \\
\hline Body length gain, $\mathrm{cm} / \mathrm{d}$ & $0.23 \pm 0.052$ & $0.30 \pm 0.052$ & $0.27 \pm 0.054$ & 0.61 \\
\hline Heart girth gain, $\mathrm{cm} / \mathrm{d}$ & $0.31 \pm 0.054$ & $0.28 \pm 0.056$ & $0.36 \pm 0.053$ & 0.99 \\
\hline Hip width gain, cm/d & $0.09 \pm 0.017$ & $0.08 \pm 0.016$ & $0.09 \pm 0.017$ & 0.92 \\
\hline ADG/total DMI, $\mathrm{kg} / \mathrm{kg}$ & $0.49 \pm 0.034$ & $0.52 \pm 0.036$ & $0.56 \pm 0.035$ & 0.35 \\
\hline \multicolumn{5}{|l|}{ After weaning (d 56-80) } \\
\hline $\mathrm{ADG}, \mathrm{kg} / \mathrm{d}$ & $1.42 \pm 0.071$ & $1.38 \pm 0.072$ & $1.35 \pm 0.075$ & 0.79 \\
\hline Withers height gain, $\mathrm{cm} / \mathrm{d}$ & $0.27 \pm 0.032$ & $0.21 \pm 0.033$ & $0.24 \pm 0.032$ & 0.51 \\
\hline Hip height gain, $\mathrm{cm} / \mathrm{d}$ & $0.23 \pm 0.040$ & $0.20 \pm 0.042$ & $0.27 \pm 0.040$ & 0.50 \\
\hline Body length gain, $\mathrm{cm} / \mathrm{d}$ & $0.34 \pm 0.062$ & $0.37 \pm 0.065$ & $0.35 \pm 0.065$ & 0.93 \\
\hline Heart girth gain, $\mathrm{cm} / \mathrm{d}$ & $0.39 \pm 0.087$ & $0.44 \pm 0.088$ & $0.32 \pm 0.091$ & 0.62 \\
\hline Hip width gain, $\mathrm{cm} / \mathrm{d}$ & $0.14 \pm 0.018$ & $0.09 \pm 0.018$ & $0.15 \pm 0.017$ & 0.11 \\
\hline ADG/total DMI, $\mathrm{kg} / \mathrm{kg}$ & $0.54 \pm 0.025$ & $0.53 \pm 0.026$ & $0.49 \pm 0.027$ & 0.36 \\
\hline
\end{tabular}

${ }^{1}$ Treatment: control $=$ calf starter containing no lactose; LAC5 $=$ calf starter containing $5 \%$ of lactose on a DM basis; LAC10 = calf starter containing $10 \%$ of lactose on a DM basis. 
Table 5. Effects of feeding calf starters differing in lactose content on fecal score $(1-4 \text { scale })^{1}$ before weaning (d 7-41), during weaning transition (d 42-55), and after weaning (d 56-80; LSM \pm SEM)

\begin{tabular}{lcccc}
\hline & \multicolumn{3}{c}{ Treatment $^{2}$} \\
\cline { 2 - 3 } & $\begin{array}{c}\text { Control } \\
(\mathrm{n}=15)\end{array}$ & $\begin{array}{c}\text { LAC5 } \\
(\mathrm{n}=14)\end{array}$ & $\begin{array}{c}\text { LAC10 } \\
(\mathrm{n}=14)\end{array}$ & $P$ value \\
\hline Item & $\begin{array}{l}1.7 \pm 0.06 \\
\text { Before weaning (d 7-41) }\end{array}$ & $1.6 \pm 0.07$ & $1.8 \pm 0.07$ & 0.11 \\
Weaning transition (d 42-55) & $1.3 \pm 0.13$ & $1.2 \pm 0.06$ & $1.3 \pm 0.05$ & 0.20 \\
After weaning (d 56-80) & $1.3 \pm 0.13$ & $1.3 \pm 0.14$ & 0.56 \\
\hline${ }^{1}$ Fecal score: 1 = normal fecal consistency to 4 = severe diarrhea. & \\
${ }^{2}$ Treatment: control = calf starter containing no lactose; LAC5 = calf starter containing 5\% of lactose on a DM \\
basis; LAC10 = calf starter containing 10\% of lactose on a DM basis.
\end{tabular}

with production of acetate or propionate (Owens and Goetsch, 1988), we expected greater butyrate concentration in ruminal fluid to be associated with higher ruminal $\mathrm{pH}$. However, we did not detect any treatment effects on ruminal butyrate concentration, ruminal $\mathrm{pH}$, calf starter intake, and growth performance. The lack of treatment effects on intake and growth performance can be attributed to similar ruminal $\mathrm{pH}$ among treatments.

\section{Ruminal $\mathrm{pH}$}

Although ruminal $\mathrm{pH}$ was not affected by treatment in the current study, it is noteworthy that lactose intake was positively correlated with daily minimum ruminal $\mathrm{pH}$, and tended to be negatively correlated with the severity of SARA indicated by area $\mathrm{pH}<5.8$. We speculated that the variation in hay intake among calves, regardless of treatment, might have masked possible positive effects of lactose on ruminal $\mathrm{pH}$. Numerous studies reported that hay intake increased ruminal $\mathrm{pH}$ of calves fed either pelleted or texturized starters (Quigley et al., 1992b; Khan et al., 2011; Laarman and Oba, 2011; Castells et al., 2013; Kim et al., 2016). We tried to minimize confounding effects of variable hay intake on treatment effects by limiting hay intake to $150 \mathrm{~g} / \mathrm{d}$; however, a small difference in hay intake can exert large effects on ruminal pH. Terré et al. (2013) reported that postweaning calves consuming a pelleted starter with $81.5 \mathrm{~g} / \mathrm{d}$ of oat hay had higher ruminal $\mathrm{pH}$ than those consumed no hay. In the current study, hay intake of calves varied from 0 to $139.4 \mathrm{~g} / \mathrm{d}$ and was

Table 6. Effects of feeding calf starters differing in lactose content on ruminal $\mathrm{pH}$ of calves immediately after weaning (d 55-62) and 3 wk after weaning (d 77-80; LSM \pm SEM)

\begin{tabular}{|c|c|c|c|c|}
\hline \multirow[b]{2}{*}{ Item } & \multicolumn{3}{|c|}{ Treatment $^{1}$} & \multirow[b]{2}{*}{$P$ value } \\
\hline & Control & LAC5 & LAC10 & \\
\hline \multicolumn{5}{|l|}{ Immediately after weaning $^{2}$} \\
\hline Minimum ruminal pH & $4.84 \pm 0.136$ & $5.01 \pm 0.054$ & $4.83 \pm 0.147$ & 0.57 \\
\hline Mean ruminal pH & $5.69 \pm 0.131$ & $5.63 \pm 0.131$ & $5.42 \pm 0.144$ & 0.37 \\
\hline Maximum ruminal pH & $7.00 \pm 0.240$ & $6.75 \pm 0.240$ & $6.29 \pm 0.262$ & 0.17 \\
\hline Duration $\mathrm{pH}<5.8{ }^{3} \mathrm{~min} / \mathrm{d}$ & $929 \pm 146.2$ & $965 \pm 146.2$ & $1,258 \pm 163.8$ & 0.31 \\
\hline Area $\mathrm{pH}<5.8,{ }^{4} \mathrm{pH} \times \min / \mathrm{d}$ & $350 \pm 129.1$ & $398 \pm 129.1$ & $585 \pm 144.0$ & 0.48 \\
\hline Daily SD & $0.20 \pm 0.229$ & $0.18 \pm 0.229$ & $0.13 \pm 0.035$ & 0.34 \\
\hline \multicolumn{5}{|l|}{3 wk after weaning ${ }^{2}$} \\
\hline Minimum ruminal $\mathrm{pH}$ & $4.85 \pm 0.114$ & $5.07 \pm 0.116$ & $5.09 \pm 0.114$ & 0.57 \\
\hline Mean ruminal pH & $5.59 \pm 0.114$ & $5.71 \pm 0.115$ & $5.74 \pm 0.115$ & 0.37 \\
\hline Maximum ruminal pH & $6.61 \pm 0.134$ & $6.64 \pm 0.136$ & $6.68 \pm 0.136$ & 0.17 \\
\hline Duration $\mathrm{pH}<5.8{ }^{3} \mathrm{~min} / \mathrm{d}$ & $1046 \pm 116.3$ & $868 \pm 125.0$ & $783 \pm 121.0$ & 0.31 \\
\hline Area $\mathrm{pH}<5.8,{ }^{4} \mathrm{pH} \times \min / \mathrm{d}$ & $460 \pm 102.8$ & $451 \pm 110.7$ & $349 \pm 106.7$ & 0.48 \\
\hline Daily SD & $0.21 \pm 0.030$ & $0.23 \pm 0.029$ & $0.19 \pm 0.029$ & 0.73 \\
\hline
\end{tabular}

${ }^{1}$ Treatment: control $(\mathrm{n}=5$ and 14 immediately and $3 \mathrm{wk}$ after weaning, respectively) $=$ calf starter containing no lactose; LAC5 ( $\mathrm{n}=5$ and 14 immediately and $3 \mathrm{wk}$ after weaning, respectively) calf starter containing $5 \%$ of lactose on a DM basis; LAC10 ( $\mathrm{n}=4$ and 14 immediately and 3 wk after weaning, respectively $)=$ calf starter containing $10 \%$ of lactose on a DM basis.

${ }^{2}$ Some $\mathrm{pH}$ data were missing due to $\mathrm{pH}$ system failures.

${ }^{3} \mathrm{pH}$ was measured every 2 min. If ruminal $\mathrm{pH}$ was below $\mathrm{pH} 5.8$, ruminal $\mathrm{pH}$ was considered to be below $\mathrm{pH}$ 5.8 for the following $2 \mathrm{~min}$.

${ }^{4}$ Area under the curve was calculated by multiplying the time ruminal $\mathrm{pH}$ was below 5.8 by $\mathrm{pH}$ units below pH 5.8 at each measurement point. 
Table 7. Spearman's correlation coefficient $\left(r_{s}\right)$ between ruminal $\mathrm{pH}$ variables and intake variables for $\mathrm{d} 77$ to 80

\begin{tabular}{lccccc}
\hline Item & $\begin{array}{c}\text { Ruminal pH } \\
(\text { maximum })\end{array}$ & $\begin{array}{c}\text { Ruminal } \mathrm{pH} \\
(\text { mean })\end{array}$ & $\begin{array}{c}\text { Ruminal } \mathrm{pH} \\
(\text { minimum })\end{array}$ & $\begin{array}{c}\text { Duration } \\
\mathrm{pH}<5.8,{ }^{1} \mathrm{~min} / \mathrm{d}\end{array}$ & $\begin{array}{c}\text { Area } \\
\mathrm{pH}<5.8,{ }^{2} \mathrm{pH} \times \mathrm{min} / \mathrm{d}\end{array}$ \\
\hline DMI, g/d & 0.042 & -0.022 & -0.055 & -0.022 & -0.032 \\
Starter intake, g/d & -0.005 & -0.074 & -0.068 & 0.018 & 0.095 \\
Hay intake, g/d & $0.408^{*}$ & $0.338^{*}$ & 0.134 & $-0.329^{*}$ & $-0.325^{*}$ \\
Starch intake, g/d & -0.075 & -0.209 & -0.238 & 0.158 & 0.184 \\
Lactose intake, g/d & 0.086 & 0.221 & $0.306^{*}$ & -0.188 & $-0.268 \dagger$ \\
\hline
\end{tabular}

${ }^{1} \mathrm{pH}$ was measured every 2 min. If ruminal $\mathrm{pH}$ was below $\mathrm{pH} 5.8$, ruminal $\mathrm{pH}$ was considered to be below $\mathrm{pH} 5.8$ for the following 2 min.

${ }^{2}$ Area under the curve was calculated by multiplying the time ruminal $\mathrm{pH}$ was below 5.8 by $\mathrm{pH}$ units below pH 5.8 at each measurement point.

$* P<0.05 ; \nmid P<0.10$.

positively correlated with dairy mean and maximum ruminal $\mathrm{pH}$ and negatively correlated with duration $\mathrm{pH}$ $<5.8$ and area $\mathrm{pH}<5.8$ at $3 \mathrm{wk}$ after weaning. Laarman et al. (2012) reported that decreasing starch content of texturized calf starters, containing $29.6 \%$ flaked grains on a DM basis, did not prevent SARA, whereas hay intake was positively correlated with mean ruminal $\mathrm{pH}$. Therefore, hay intake may influence ruminal fermentation to a greater extent than carbohydrate profiles or starch content of calf starters differing in their physical form.

The lack of treatment effects on ruminal $\mathrm{pH}$ might be also attributed to the feeding method of calf starters in the current study. We offered calf starters twice daily, and they were always available to calves. As such, calves did not have a large meal immediately after feed- ing (personal observation; data not shown), and the reduction in postprandial ruminal $\mathrm{pH}$ was less than 0.3 units (Figure 1). As the control calf starter did not cause severe SARA, treatment starters that partially replaced dietary starch with lactose might not have affected overall ruminal $\mathrm{pH}$, although lactose intake was positively correlated with daily minimum ruminal $\mathrm{pH}$. The lack of drastic reduction in postprandial ruminal $\mathrm{pH}$ in the current study is contrary to the report of Laarman et al. (2012), who observed more than a 1.0 unit reduction in ruminal $\mathrm{pH}$ within $3 \mathrm{~h}$ after feeding. Both Laarman et al. (2012) and our current study used texturized calf starters containing approximately $30 \%$ of flaked grains on a DM basis. However, in the study of Laarman et al. (2012), calves were offered starters once daily, and its amount was restricted to $2.5 \mathrm{~kg} / \mathrm{d}$. Limit-

Table 8. Effects of feeding calf starters differing in lactose content on ruminal VFA concentration and profile in calves 1 wk after weaning (d $62)$ and after 3 wk after weaning (d 80; LSM \pm SEM)

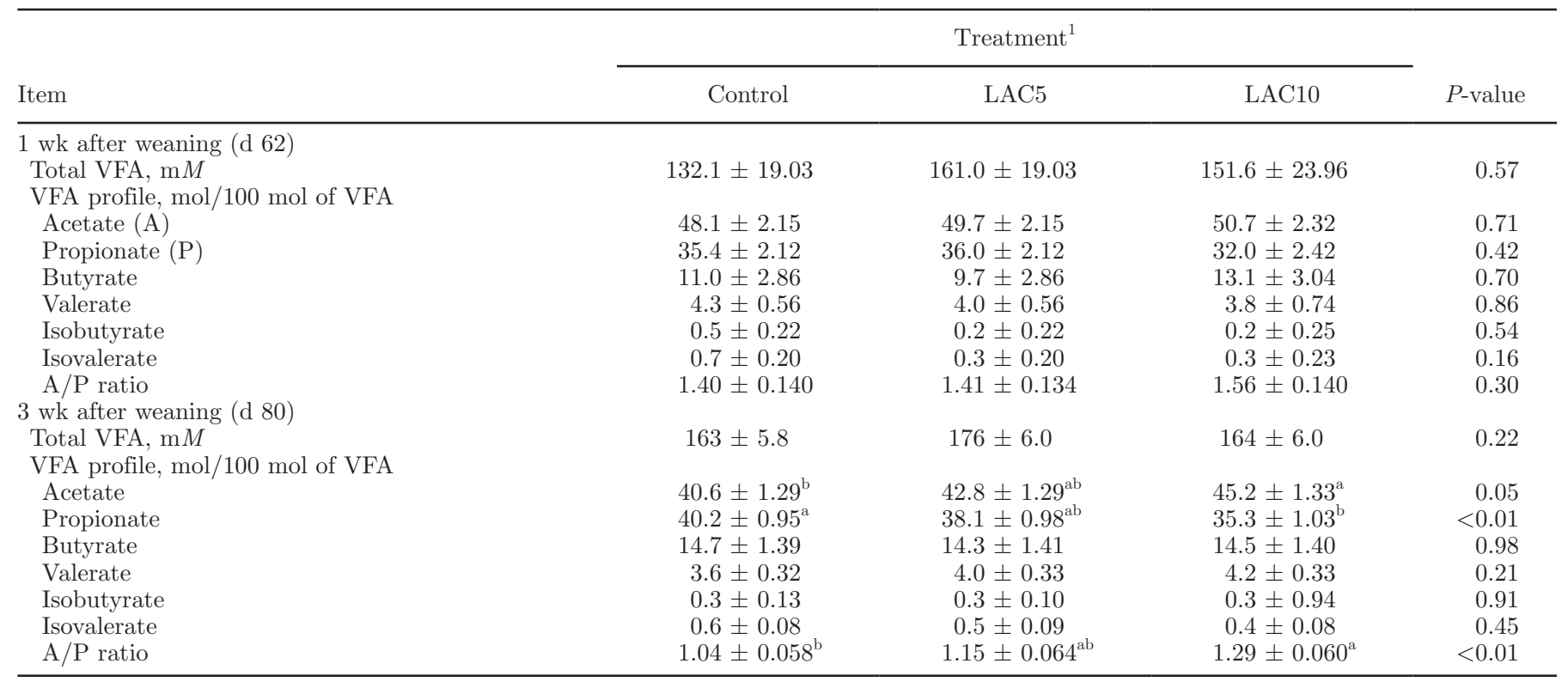

${ }^{\mathrm{a}-\mathrm{c}}$ Means within a row differ $(P<0.05)$ if superscript letters differ.

${ }^{1}$ Treatment: control $(\mathrm{n}=5$ and 15 immediately and $3 \mathrm{wk}$ after weaning, respectively) $=$ calf starter containing no lactose; LAC5 $(\mathrm{n}=5$ and 14 immediately and $3 \mathrm{wk}$ after weaning, respectively) = calf starter containing $5 \%$ of lactose on a DM basis; LAC10 (n = 5 and 14 immediately and 3 wk after weaning, respectively) = calf starter containing $10 \%$ of lactose on a DM basis. 


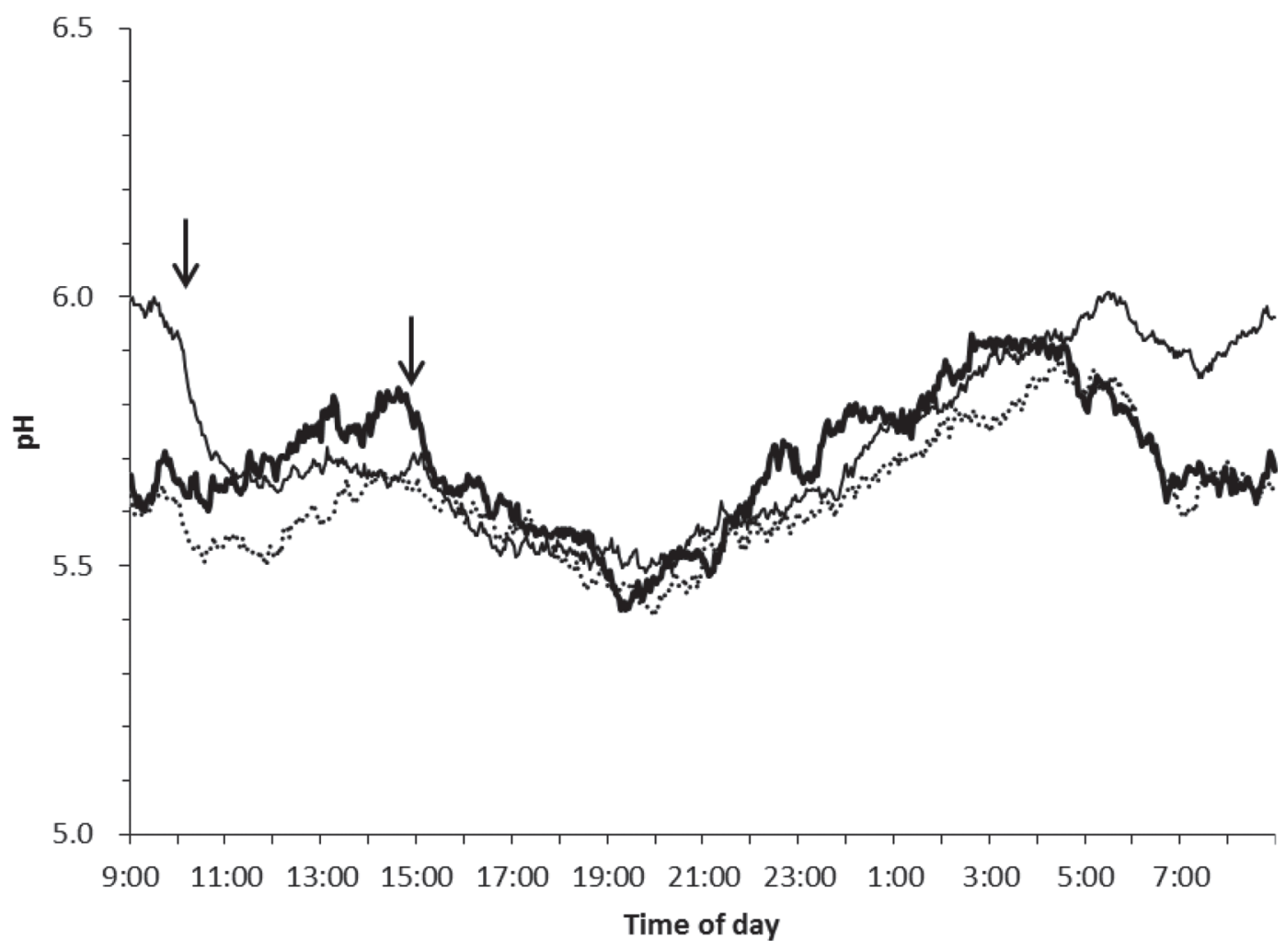

Figure 1. Effects of feeding calf starters differing in lactose content on diurnal changes in ruminal $\mathrm{pH}$ on d 77 to 80 . Control $=$ starter containing no lactose (dashed line; $\mathrm{n}=13$ ); LAC5 = starter containing $5 \%$ lactose on a DM basis (thin solid line; $\mathrm{n}=14$ ); LAC10 = starter containing $10 \%$ lactose on a DM basis (thick solid line; $\mathrm{n}=14$ ). Calves were fed treatment calf starters twice daily at 1000 and $1500 \mathrm{~h}$, as incidated by arrows.

feeding calf starters, to avoid excess fermentation in the rumen, may have induced slug-feeding and resulted in SARA (Krause and Oetzel, 2006; Kitts et al., 2011), as slug-feeding causes drastic reduction in ruminal $\mathrm{pH}$ after feeding in mature cows (Stone, 2004; Krause and Oetzel, 2006) and calves (Quigley et al., 1992b). These results indicate that feeding methods and the consequent eating pattern of calves would exert substantial effects on ruminal $\mathrm{pH}$, potentially masking effects of feeding calf starters differing in starch content.

\section{Ruminal VFA Profile}

We had hypothesized that lactose inclusion in calf starters would increase ruminal butyrate concentration, but we did not observe treatment effects on butyrate concentration. Contrarily, the LAC10 treatment increased the molar ratio of acetate and decreased molar ratio of propionate in ruminal fluid, resulting in a higher acetate-to-propionate ratio compared with control at 3 wk after weaning. These results might be at least partially attributed to differences in NDF intake among treatments; NDF intake was greater for LAC10 compared with control partly due to the greater NDF content of LAC10 calf starter, although it was not intended. Our observation is consistent with Suárez et al. (2006a), who reported higher NDF intake was associated with greater acetate-to-propionate ratio.

Our finding that lactose inclusion in calf starters increased ruminal concentration of acetate, instead of butyrate, is contrary to previous research findings with mature cows (DeFrain et al., 2004; DeFrain et al., 2006; Oba et al., 2015). Responses to lactose feeding in calves might be different from those in mature cows. In general, forage intake is much higher in mature cows compared with calves (Drackley, 2008; Khan et al., 2016), resulting in high acetate production in the rumen (Sutton et al., 2003). Greater acetate production is generally associated with greater metabolic hydrogen production in the rumen (Janssen, 2010), but its accumulation in the rumen is not favorable (Wolin et al., 1997; Hegarty and Gerdes, 1999). Glucose fermentation to butyrate generates half as much metabolic hydrogen as that to acetate (France and Dijkstra 2005; Ungerfeld and Kohn, 2008). As such, if feeding lactose increases butyrate production (DeFrain et al., 2004, 2006; Oba et al., 2015), accumulation of metabolic hydrogen in the rumen can be reduced. However, calves before or 
right after weaning consumed highly fermentable carbohydrates compared with mature cows (Baldwin et al., 2004; Drackley, 2008). Consumption of calf starters high in starch content is associated with greater propionate production (Žitnan et al., 1998; Lesmeister and Heinrichs, 2004; Kristensen et al., 2007), which decreases metabolic hydrogen concentration in the rumen (Janssen, 2010) as the metabolic pathway to produce propionate consumes metabolic hydrogen (France and Dijkstra 2005; Ungerfeld and Kohn, 2008). As a consequence, ruminal hydrogen concentration might be low in the rumen of calves, and this could allow more acetate production, as it is favored at low metabolic hydrogen concentrations (Hegarty and Gerdes, 1999; Janssen, 2010). In addition, production of acetate yields more ATP than butyrate (France and Dijkstra, 2005; Ungerfeld and Kohn, 2008) and is energetically more advantageous to ruminal microbes. However, we did not measure ruminal metabolic hydrogen concentration in the current study; further investigation is warranted to evaluate the speculations.

Although it is speculated that effects of lactose on ruminal VFA profile would differ between mature cows and calves, rapid fermentation of lactose along with fast absorption of butyrate should not be excluded as a possible reason explaining the lack of treatment effects on ruminal butyrate concentration. Absorption of butyrate can be stimulated at low ruminal $\mathrm{pH}$; fractional absorption rate of butyrate was higher than acetate at $\mathrm{pH} 5.4$ (Dijkstra, 1994) and in calves with ruminal $\mathrm{pH}$ below 6.0 (Weigand et al., 1972). In the current study, mean ruminal $\mathrm{pH}$ ranged from 5.42 to 5.69 , which makes it possible to alter absorption rate of butyrate. In addition, it is reported that increasing ruminal infusion of VFA did not affect ruminal concentration of butyrate although it increased acetate concentration (Dijkstra et al., 1994; López et al., 2003). As such, butyrate concentration in ruminal fluid may not necessarily reflect butyrate production in the rumen, and it is possible to increase butyrate production in the rumen without affecting its concentration.

\section{CONCLUSIONS}

Lactose inclusion in calf starter up to $10 \%$ of DM did not affect ruminal $\mathrm{pH}$, DMI, or growth performance. However, lactose intake was positively correlated with minimum ruminal $\mathrm{pH}$, and we cannot exclude the possibility that partial replacement of dietary starch with lactose increases ruminal $\mathrm{pH}$. It should be noted that hay intake, feeding method of calf starter, and feeding pattern of calves could exert substantial effects on ruminal $\mathrm{pH}$ in calves during the weaning transition, and these management factors require as much attention as nutrient composition of calf starters to avoid SARA in calves. In addition, we observed that lactose inclusion in calf starters increased acetate concentration in the rumen without affecting butyrate concentration. Effects of feeding lactose on ruminal VFA may be different between mature cows and calves or depending on the basal diets that animals consume, and this requires further investigation.

\section{REFERENCES}

Anderson, K. L., T. G. Nagaraja, and J. L. Morrill. 1987. Ruminal metabolic development in claves weand conventionally or early. J. Dairy Sci. 70:1000-1005.

AOAC. 1990. Official Methods of Analysis. 15th ed. Assoc. Off. Anal. Chem, Gaithersburg, MD.

AOAC International. 2002. Official Methods of Analysis. 17th ed. AOAC International, Gaithersburg, MD.

Baldwin, R. L. V., K. R. McLeod, J. L. Klotz, and R. N. Heitmann. 2004. Rumen development, intestinal growth and hepatic metabolism in the pre-and postweaning ruminant. J. Dairy Sci. 87(Suppl.):E55-E65.

Bergman, E. N. 1990. Energy contributions of volatile fatty acids from the gastrointestinal tract in various species. Physiol. Rev. 70:567-590.

Bull, L. S., L. J. Bush, J. D. Friend, B. Harris Jr., and E. W. Jones. 1965. Incidence of ruminal parakeratosis in calves fed different rations and its relation to volatile fatty acid absorption. J. Dairy Sci. 48:1459-1466.

Castells, L., A. Bach, A. Aris, and M. Terré. 2013. Effects of forage provision to young calves on rumen fermentation and development of the gastrointestinal tract. J. Dairy Sci. 96:5226-5236.

Chamberlain, D. G., S. Robertson, and J. Choung. 1993. Sugars versus starch as supplements to grass silage: Effects on ruminal fermentation and the supply of microbial protein to the small intestine, estimated from the urinary excretion of purine derivatives, in sheep. J. Sci. Food Agric. 63:189-194.

DeFrain, J. M., A. R. Hippen, K. F. Kalscheur, and D. J. Schingoethe. 2004. Feeding lactose increases ruminal butyrate and plasma betahydroxybutyrate in lactating dairy cows. J. Dairy Sci. 87:24862494.

DeFrain, J. M., A. R. Hippen, K. F. Kalscheur, and D. J. Schingoethe. 2006. Feeding lactose to increase ruminal butyrate and the metabolic status of transition dairy cows. J. Dairy Sci. 89:267-276.

Dijkstra, J. 1994. Production and absorption of volatile fatty acids in the rumen. Livest. Prod. Sci. 39:61-69.

Drackley, J. K. 2008. Calf nutrition from birth to breeding. Vet. Clin. North Am. Food Anim. Pract. 24:55-86.

France, J., and J. Dijkstra. 2005. Volatile fatty acid production. Pages 157-175 in Quantitative Aspects of Ruminant Digestion and Metabolism. 2nd ed. J. Dijkstra, J. M. Forbes, and J. France, ed. CABI Publishing, Cambridge, MA.

Garret, E. 1996. Subacute rumen acidosis - Clinical signs and diagnosis in dairy herds. Large Anim. Vet. 11:6-10.

Hall, M. B., W. H. Hoover, J. P. Jennings, and T. K. Miller Webster. 1999. A method for partitioning neutral detergent soluble carbohydrates. J. Sci. Food Agric. 79:2079-2086.

Hegarty, R. S., and R. Gerdes. 1999. Hydrogen production and transfer in the rumen. Recent Adv. Anim. Nutr. Aust. 12:37-44.

Inabu, Y., A. Saegusa, K. Inouchi, M. Oba, and T. Sugino. 2016. Plasma concentrations of glucagon-like peptide 1 and 2 in calves fed calf starters containing lactose. J. Dairy Sci. 99(E. Suppl. 1):539.

Janssen, P. H. 2010. Influence of hydrogen on rumen methane formation and fermentation balances through microbial growth kinetics and fermentation thermodynamics. Anim. Feed Sci. Technol. 160:1-22.

Kay, R. N. B. 1960. The rate of flow and composition of various salivary secretions in sheep and calves. J. Physiol. 150:515-537. 
Khan, M. A., A. Bach, D. M. Weary, and M. A. G. von Keyserlingk. 2016. Transitioning from milk to solid feed in dairy heifers. J. Dairy Sci. 99:885-902.

Khan, M. A., H. J. Lee, W. S. Lee, H. S. Kim, S. B. Kim, S. B. Park, K. S. Baek, J. K. Ha, and Y. J. Choi. 2008. Starch source evaluation in calf starter: II. Ruminal parameters, rumen development, nutrient digestibilities, and nitrogen utilization in Holstein calves. J. Dairy Sci. 91:1140-1149.

Khan, M. A., D. M. Weary, and M. A. G. von Keyserlingk. 2011. Hay intake improves performance and rumen development of calves fed higher quantities of milk. J. Dairy Sci. 94:3547-3553.

Kim, Y. H., N. Touji, K. Kizaki, S. Kushibiki, T. Ichijo, and S. Sato. 2016. Effects of dietary forage and calf starter on ruminal $\mathrm{pH}$ and transcriptomic adaptation of the rumen epithelium in Holstein calves during the weaning transition. Physiol. Genomics 48:803-809.

Kitts, B. L., J. J. Duncan, B. W. McBride, and T. J. Devries. 2011. Effect of the provision of a low-nutritive feedstuff on the behavior of dairy heifers limit fed a high-concentrate ration. J. Dairy Sci. 94:940-950.

Kleen, J. L., G. A. Hooijer, J. Rehage, and J. P. Noordhuizen. 2003 Subacute ruminal acidosis (SARA): A review. J. Vet. Med. A Physiol. Pathol. Clin. Med. 50:406-414.

Krause, K. M., and G. R. Oetzel. 2006. Understanding and preventing subacute ruminal acidosis in dairy herds: A review. Anim. Feed Sci. Technol. 126:215-236.

Kristensen, N. B., J. Sehested, S. K. Jensen, and M. Vestergaard. 2007. Effect of milk allowance on concentrate intake, ruminal environment, and ruminal development in milk-fed Holstein calves. J. Dairy Sci. 90:4346-4355.

Laarman, A. H., and M. Oba. 2011. Effect of calf starter on rumen $\mathrm{pH}$ of Holstein dairy calves at weaning. J. Dairy Sci. 94:5661-5664.

Laarman, A. H., T. Sugino, and M. Oba. 2012. Effects of starch content of calf starter on growth and rumen $\mathrm{pH}$ in Holstein calves during the weaning transition. J. Dairy Sci. 95:4478-4487.

Lesmeister, K. E., and A. J. Heinrichs. 2004. Effects of corn processing on growth characteristics, rumen development, and rumen parameters in neonatal dairy calves. J. Dairy Sci. 87:3439-3450.

López, S., F. D. D. Hovell, J. Dijkstra, and J. France. 2003. Effects of volatile fatty acid supply on their absorption and on water kinetics in the rumen of sheep sustained by intragastric infusions. J. Anim. Sci. 81:2609-2616.

Nocek, J. E., C. W. Heald, and C. E. Polan. 1984. Influence of ration physical form and nitrogen availability on ruminal morphology of growing bull calves. J. Dairy Sci. 67:334-343.

Government of Japan. 2015. Nutrition Labeling Standards. Consumer Affairs Agency, Government of Japan, Tokyo.

Oba, M., J. L. Mewis, and Z. Zhining. 2015. Effects of ruminal doses of sucrose, lactose, and corn starch on ruminal fermentation and expression of genes in ruminal epithelial cells. J. Dairy Sci. 98:586-594.

Owens, F. N., and A. L. Goetsch. 1988. Ruminal fermentation. Pages 145-171 in The Ruminant Animal: Digestive Physiology and Nutrition. D. C. Church, ed. Prentice-Hall, Englewood Cliffs, NJ.

Quigley, J. D. III, S. I. Boehms, T. M. Steen, and R. N. Heitmann. 1992a. Effect of lasalocid on selected ruminal and blood metabolites in young calves. J. Dairy Sci. 75:2235-2241.
Quigley, J. D. III, T. M. Steen, and S. I. Boehms. 1992b. Postprandial changes of selected blood and ruminal metabolites in ruminating calves fed diets with or without hay. J. Dairy Sci. 75:228-235.

Stone, W. C. 2004. Nutritional approaches to minimize subacute, ruminal acidosis and laminitis in dairy cattle. J. Dairy Sci. 87:E13E26.

Suárez, B. J., C. G. Van Reenen, G. Beldman, J. van Delen, J. Dijkstra, and W. J. J. Gerrits. 2006a. Effects of supplementing concentrates differing in carbohydrate composition in veal calf diets: I. Animal performance and rumen fermentation characteristics. J. Dairy Sci. 89:4365-4375.

Suárez, B. J., C. G. Van Reenen, W. J. J. Gerrits, N. Stockhofe, A. M. van Vuuren, and J. Dijkstra. 2006b. Effects of supplementing concentrates differing in carbohydrate composition in veal calf diets: II. rumen development. J. Dairy Sci. 89:4376-4386.

Sutton, J. D., M. S. Dhanoa, S. V. Morant, J. France, D. J. Napper, and E. Schuller. 2003. Rates of production of acetate, propionate, and butyrate in the rumen of lactating dairy cows given normal and low-roughage diets. J. Dairy Sci. 86:3620-3633.

Tamate, H., A. D. McGilliard, N. L. Jacobson, and R. Getty. 1962 Effect of various dietaries on the anatomical development of the stomach in the calf. J. Dairy Sci. 45:408-420.

Terré, M., E. Pedrals, A. Dalmau, and A. Bach. 2013. What do preweaned and weaned calves need in the diet: A high fiber content or a forage source? J. Dairy Sci. 96:5217-5225.

Ungerfeld, E. M., and R. A. Kohn. 2008. The role of thermodynamics in the control of ruminal fermentation. Pages 159-170 in Ruminant Physiology: Digestion, Metabolism and Impact of Nutrition on Gene Expression, Immunology and Stress. K. Sejrsen, T. Hvelplund, and M. O. Nielsen, ed. Wageningen Academic Publishers, Wageningen, the Netherlands.

Watanabe, Y., R. Suzuki, S. Koike, K. Nagashima, M. Mochizuki, R. J. Forster, and Y. Kobayashi. 2010. In vitro evaluation of cashew nut shell liquid as a methane-inhibiting and propionate-enhancing agent for ruminants. J. Dairy Sci. 93:5258-5267.

Weigand, E., J. W. Young, and A. D. McGilliard. 1972. Extent of butyrate metabolism by bovine ruminoreticulum epithelium and the relationship to absorption rate. J. Dairy Sci. 55:589-597.

Williams, P. E. V., R. J. Fallon, G. M. Innes, and P. Garthwaite. 1987. Effect on food intake, rumen development and live weight of calves of replacing barley with sugar beet-citrus pulp in a starter diet. Anim. Prod. 44:65-73.

Wolin, M. J., T. L. Miller, and C. S. Stewart. 1997. Microbe-microbe interactions. Pages 467-431 in The Rumen Microbial Ecosystem. P. N. Hobson and C. S. Stewart, ed. Blackie Academic \& Professional, London, UK.

Yáñez-Ruiz, D. R., L. Abecia, and C. J. Newbold. 2015. Manipulating rumen microbiome and fermentation through interventions during early life: a review. Front. Microbiol. 6:1133.

Žitnan, R., J. Voigt, U. Schonhusen, J. Wegner, M. Kokardova, H. Hagemeister, M. Levkut, S. Kuhla, and A. Sommer. 1998. Influence of dietary concentrate to forage ratio on the development of rumen mucosa in calves. Arch. Tierernahr. 51:279-291. 